\title{
Prospects for precision measurements on ammonia molecules in a fountain
}

\author{
H.L. Bethlem ${ }^{1,2, a}$, M. Kajita ${ }^{3}$, B. Sartakov ${ }^{4}$, G. Meijer ${ }^{2}$, and W. Ubachs ${ }^{1}$ \\ 1 Laser Centre Vrije Universiteit, De Boelelaan 1081, 1081HV Amsterdam, The Netherlands \\ 2 Fritz-Haber-Institut der Max-Planck-Gesellschaft, Faradayweg 4-6, 14195 Berlin, Germany \\ 3 National Institute of Information and Communications Technology, Nukui-Kitamachi, \\ Koganei, Tokyo 184-8795, Japan \\ ${ }^{4}$ General Physics Institute RAS, Vavilov Str. 38, 119991 Moscow, Russia
}

\begin{abstract}
The recent demonstration of cooling and manipulation techniques for molecules offer new possibilities for precision measurements in molecules. Here, we present the design of a molecular fountain based on a Stark decelerated molecular beam. In this fountain, ammonia molecules are decelerated to a few meter per second, cooled to sub microKelvin temperatures and subsequently launched. The molecules fly upwards some $30 \mathrm{~cm}$ before falling back under gravity, thereby passing a microwave cavity twice - as they fly up and as they fall back down. The effective interrogation time in such a Ramsey type measurement scheme includes the entire flight time between the two traversals through the driving field, which is on the order of a $1 / 2$ second. We present numerical simulations of the trajectories through the decelerator and estimate the expected count rate. We present an evaluation of the expected stability and accuracy for the inversion transition in ${ }^{15} \mathrm{NH}_{3}$ around $22.6 \mathrm{GHz}$. The estimated frequency instability is $7 \times 10^{-12} \tau^{-1 / 2}$, with $\tau$ being the measurement time in seconds. With a careful design of the interogation zone, systematic frequency shifts are kept below $10^{-14}$. Besides serving as a proof-of-principle, these measurements may be used as a test of the time-variation of fundamental constants using the sensitivity of the tunneling motion to a change of the proton-electron mass ratio.
\end{abstract}

\section{Introduction}

The precision and reproducibility of any experiment depends on how well experimental conditions are controlled. Control over the motion of atoms and atomic ions using laser cooling and trapping techniques has dramatically increased the precision of atomic frequency standards. An accuracy exceeding $10^{-15}$ is now routinely obtained in atomic fountains based on laser cooled atoms [1,2], in atoms trapped in optical lattices [3] and in single ions trapped in an rf quadrupole field [4]. It is expected that the emerging techniques to cool and manipulate molecules $[5,6]$ will have a similarly dramatic impact on the precision of frequency measurements in molecules. One promising molecular cooling technique exploits the fact that a polar molecule experiences a force in an inhomogeneous electric field. Although this force is typically some 8-10 orders of magnitude smaller than the force that can be applied to charged particles, it is nonetheless sufficient to achieve complete control over polar molecules. Recently, high resolution microwave spectra of $\mathrm{ND}_{3}$ [7] and $\mathrm{OH}$ [8] molecules have been recorded on Stark decelerated beams, demonstrating the potential of cold molecules. In this paper, we present the design of a molecular fountain based on a Stark decelerated ammonia beam that is currently

\footnotetext{
a e-mail: rick@few.vu.nl
} 
under construction at the Laser Centre Vrije Universiteit and evaluate the accuracy that might be obtained in this fountain.

In the near future, it is unlikely that the precision obtained in molecular fountains will be able to compete with that obtained in their atomic counterparts. The merits of a molecular fountain lie in the possibility it offers for precision tests of fundamental physics theories. The sensitivity of any experiment looking for a frequency shift due to a certain physical phenomenom depends both on the size of the shift, i.e., the inherent sensitivity of the atom or molecule to a certain effect, and on the ability to measure this shift. The structure (and symmetry) of molecules makes them inherently more sensitive for a number of tests. For instance, in certain molecules like $\mathrm{YbF}$ and $\mathrm{PbO}$, time-symmetry violating interactions leading to a permanent electric dipole moment are three orders of magnitude stronger than in atoms $[9,10]$. Molecules are also used in the study of weak interactions leading to an anapole moment of the nucleus [11], in the search for a difference in transition frequency between chiral molecules that are each other's mirror-image [12], and for constraining a possible variation of the proton-electron mass ratio $[13,14]$. The demonstration of a fountain will be relevant for all these experiments.

In principle, the fountain may be used to study transitions in many polar molecules. We plan to use a specific isotopomer of ammonia, ${ }^{15} \mathrm{NH}_{3}$, as test molecule for a number of reasons: (i) The microwave spectrum of ammonia is very well studied. In fact, it was used in the first demonstration of an atomic clock by Condon and Lyons in 1948 [15] and the first demonstration of the MASER by Gordon and co-workers in 1955 [16]. The energies of the hyperfine levels of the four most common symmetric isotopomers $\left({ }^{14} \mathrm{NH}_{3},{ }^{15} \mathrm{NH}_{3},{ }^{14} \mathrm{ND}_{3}\right.$ and $\left.{ }^{15} \mathrm{ND}_{3}\right)$ have been determined with an accuracy of better than $100 \mathrm{~Hz}$ [7,17-19]. We choose $\mathrm{NH}_{3}$ over $\mathrm{ND}_{3}$ because the inversion frequency is higher and because $\mathrm{NH}_{3}$ has a more convenient Stark shift. We choose ${ }^{15} \mathrm{NH}_{3}$ over ${ }^{14} \mathrm{NH}_{3}$ because it has a less complicated hyperfine structure. (ii) Ammonia has a strong Stark shift, a relatively small mass and a high vapour pressure. Together, these properties make ammonia very suitable for Stark deceleration. (iii) The ammonia molecule is a sensitive probe for variations of the proton-electron mass ratio. The inversion frequency in ammonia is determined by the tunneling rate of the protons through the barrier between the two equivalent configurations of the molecule and is exponentially dependent on the proton mass. By monitoring the inversion frequency over a period of a few years, a possible variation of the proton-electron mass ratio can be constrained or measured.

We will first discuss the level structure and Stark shift in ammonia. In section 3, the layout of the fountain is presented together with numerical simulations of trajectories of ammonia molecules through the fountain. From these simulations, we estimate the number of detected molecules per fountain cycle. In section 4 , we estimate the stability and evaluate all known contributions to frequency uncertainty in the inversion transition in ${ }^{15} \mathrm{NH}_{3}$ around $22.6 \mathrm{GHz}$. Finally, in section 5, we will determine the sensitivity of the inversion frequency to a variation of the proton-electron mass ratio.

\section{Energy level structure and Stark shift in ammonia}

In the electronic ground state, ammonia has a pyramidal structure with the three hydrogen atoms on a plane and the nitrogen atom at the apex. Fig. 1 shows the potential energy as the function of the umbrella angle; the angle between the molecular symmetry axis and a line connecting the nitrogen atom with one of the hydrogen atoms. Note that in the BornOppenheimer approximation this potential curve is identical for all isotopomers of ammonia. The horizontal lines show the energy of the $\nu_{2}$ umbrella vibration in the double well. If the barrier were infinitely high, the lowest two vibrational levels would be degenerate. As the barrier is finite, the nitrogen atom can tunnel quantum mechanically through the plane of the three $\mathrm{H}$ atoms. This tunnel motion gives rise to a splitting between the two inversion levels. In the case of ${ }^{15} \mathrm{NH}_{3}$ the splitting is $22.6 \mathrm{GHz}$. Due to the higher mass of the deuterium atoms the tunneling frequency is reduced to $1.43 \mathrm{GHz}$ in ${ }^{15} \mathrm{ND}_{3}$. This exceptional sensitivity of the inversion frequency to the reduced mass of the molecule makes ammonia very suitable for constraining a possible variation of the proton-electron mass ratio. This is discussed in detail in section 5 . 


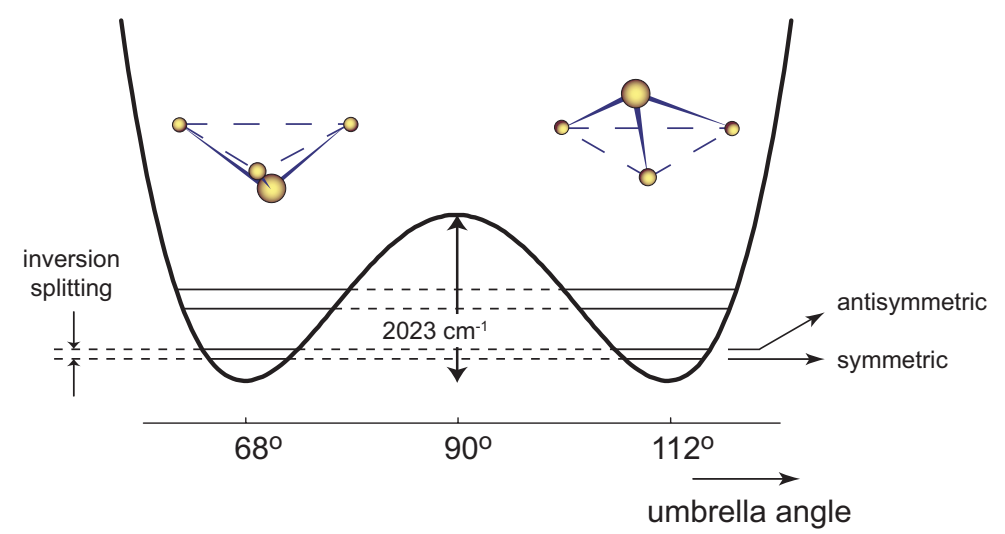

Fig. 1. Potential energy of the $\nu_{2}$ (umbrella) mode with the lowest vibrational levels. The splitting between the symmetric and antisymmetric levels in ${ }^{15} \mathrm{NH}_{3}$ is $0.75 \mathrm{~cm}^{-1}(22.6 \mathrm{GHz})$.

The rotational levels are labeled by the rotational quantum number, $J$, the projection of $\mathbf{J}$ on the $D_{3 h}$ molecular symmetry axis, $K$, and the projection of $\mathbf{J}$ on the space-fixed axis, $M_{J}$. In the absence of an electric field there is no preferred direction in space and the wave functions form symmetric and antisymmetric combinations (parity). Eigenfunctions with a definite parity can be constructed from linear combinations of $\left|J K M_{J}\right\rangle$ and $\left|J-K M_{J}\right\rangle$ states. The various ammonia isotopomers have different possible symmetries of the nuclear spin wavefunctions of the identical H/D nuclei. Each ro-vibrational level of the ammonia molecule combines with a nuclear spin wave function of one specific symmetry. The nuclear wavefunction of molecules in levels with $K=0,3,6, \ldots$ have $A$-symmetry and the nuclear wavefunction of molecules in levels with $K=1,2,4,5, \ldots$ have $E$-symmetry [20]. For historical reasons, levels of $A$-symmetry in $\mathrm{NH}_{3}$ are denoted as ortho levels and levels of $E$-symmetry are denoted as para levels. In a molecular beam, the molecules are adiabatically cooled, and only the lowest rotational levels in the vibrational and electronic ground state are populated. In this cooling process the symmetry of the nuclear spin wavefunction is preserved. Therefore, the ratio of the populations in the levels having different symmetries of the nuclear spin wavefunction in the molecular beam is the same as in the original sample. For $\mathrm{NH}_{3}$ this implies that roughly $60 \%$ of the molecules in the beam reside in the $|J, K\rangle=|1,1\rangle$ level which is the ground state of para-ammonia. The other $40 \%$ of the molecules are distributed over the $|J, K\rangle=|0,0\rangle$ and $|1,0\rangle$ levels. In our experiment, we use the $|J, K\rangle=|1,1\rangle$ level which is the only populated state with a significant Stark effect.

Each ro-vibrational level is further split due to hyperfine interactions. The hyperfine structure of the $|J, K\rangle=|1,1\rangle$ level of ${ }^{15} \mathrm{NH}_{3}$ is shown in Fig. 2. For convenience, the hyperfine levels have been given an index number running from 1 to 8 . The levels are further labelled by the quantum numbers $F_{1}$ and $I_{H}$, where $\mathbf{F}_{1}=\mathbf{J}+\mathbf{I}_{N}$, with $\mathbf{I}_{N}$ is the nitrogen nuclear-spin vector, and $\mathbf{I}_{\mathbf{H}}$ the overall nuclear-spin angular momentum vector of the three hydrogen atoms. $F_{1}$ and $I_{H}$, couple to give the only good quantum numbers $F$ and $M_{F}$. Following the notation used by Hougen [18], levels are labelled by their expectation value of $F_{1}$. Since $F_{1}$ is not a perfectly good quantum number, this expectation value does not need to have a half-integer value. In ${ }^{14} \mathrm{NH}_{3}$ the strong quadrupole interaction of the ${ }^{14} \mathrm{~N}$ nucleus causes splittings of a few $\mathrm{MHz}$ between levels of different $F_{1}$. In contrast, the quadrupole interaction of the ${ }^{15} \mathrm{~N}$ nucleus is zero and all hyperfine levels lie within $100 \mathrm{kHz}$.

Upon applying an electric field, levels of the same $M_{F}$ but different parity interact and repel each other. In Fig. 3 the energy of the $|J, K\rangle=|1,1\rangle$ level is shown in high (a) and low (b) electric fields (for details on the Stark effect in poly-atomic molecules, see, for instance, [21]). When an electric field is applied 8 of the 12 hyperfine components of the upper inversion level shift to higher energies, while 8 of the 12 hyperfine components of the lower inversion level shift to lower energies. We will be most interested in the $F^{\prime}=1, F_{1}^{\prime}=0.5$ and the $F^{\prime}=0$, $F_{1}^{\prime}=0.5$, i.e., levels 7 and 8 , which have the highest energy. These levels have no crossings 


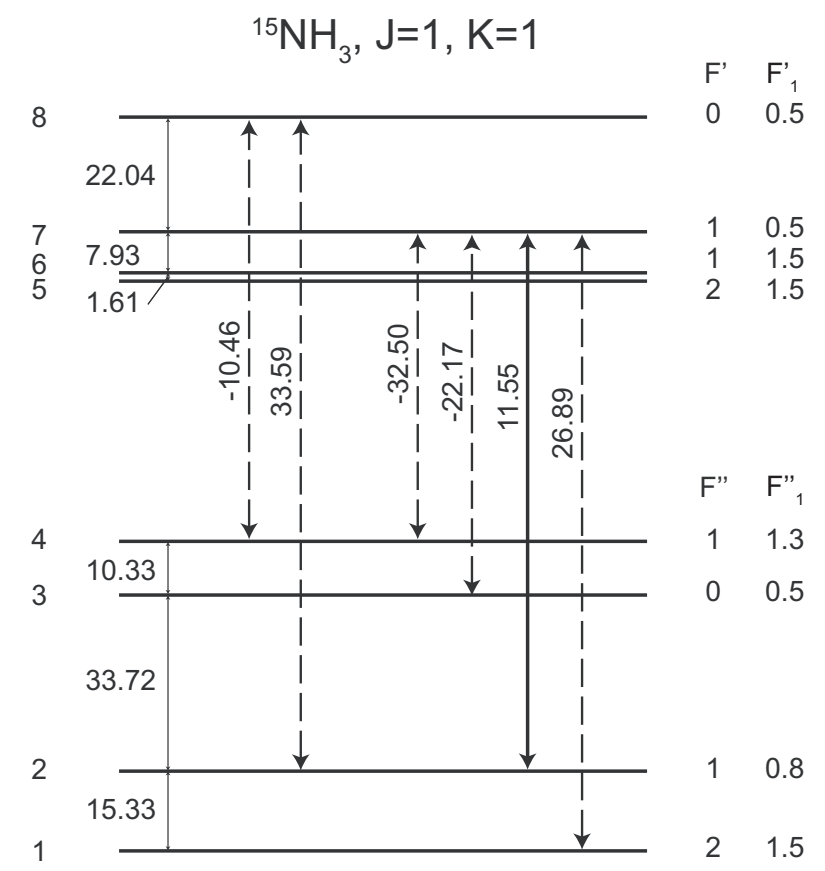

Fig. 2. Hyperfine splittings in the symmetric and antisymmetric components of the $J=1, K=1$ level of ${ }^{15} \mathrm{NH}_{3}$ taken from Hougen [18]. For convenience, the hyperfine levels have been given an index number running from 1 to 8 . The level spacings are expressed in $\mathrm{kHz}$. The arrows show possible transitions from the $F_{1}^{\prime}=0.5, F^{\prime}=0,1$ levels. The transition frequencies are in $\mathrm{kHz}$ with respect to $22624929.53 \mathrm{kHz}$. From these transitions the $F^{\prime}=1, F_{1}^{\prime}=0.5 \leftrightarrow F^{\prime \prime}=1, F_{1}^{\prime \prime}=0.8,(7 \leftrightarrow 2)$ transition at $22624941.08 \mathrm{kHz}$, indicated by the bold arrow, offers the highest accuracy.

(a)

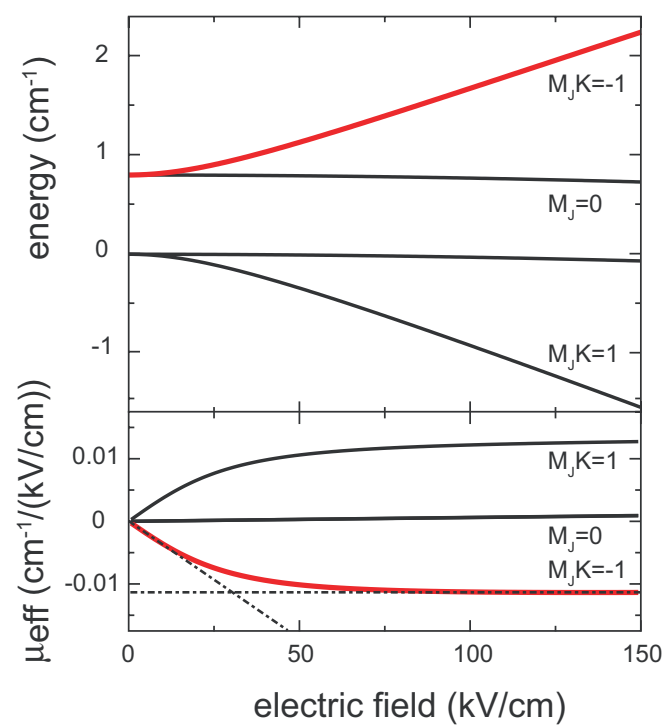

(b)

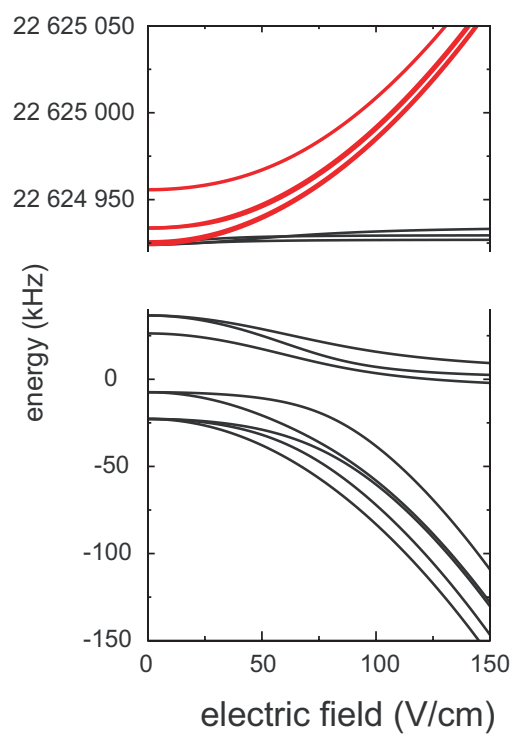

Fig. 3. (a) Stark effect and effective dipole moment for the $J=1, K=1$ state of ${ }^{15} \mathrm{NH}_{3}$ in high electric fields. $1 \mathrm{~cm}^{-1} \sim 30 \mathrm{GHz}$. The low-field seeking components are the bold red curves. (b) Stark effect for the $J=1, K=1$ state of ${ }^{15} \mathrm{NH}_{3}$ in low electric fields. 
with other hyperfine levels in electric fields so they are immune to losses due to reorientation in small electric fields.

When the Stark shift becomes larger than the hyperfine splittings, $M_{J}$ becomes a good quantum number and the hyperfine levels form four distinct groups. The distance between different hyperfine levels of the same $M_{J}$ remains constant as the electric field is further increased. When the interaction between different $J$ levels is neglected, the Stark shift of these $M_{J}$ levels is approximately given by:

$$
\Delta W_{\text {Stark }}= \pm \sqrt{\left(\frac{W_{i n v}}{2}\right)^{2}+\left(\mu|\mathbf{E}| \frac{M_{J} K}{J(J+1)}\right)^{2}} \mp \frac{W_{i n v}}{2} .
$$

Below the Stark curves the effective dipole moment, defined as

$$
\mu_{e f f}(E)=-\frac{\partial W}{\partial E}
$$

is shown as a function of electric field. For fields below $10 \mathrm{kV} / \mathrm{cm}$ the second term in Eq. (1) is much smaller than the first term and the Stark shift is approximately quadratic. For fields larger than $50 \mathrm{kV} / \mathrm{cm}$ the second term is much larger than the first term and the Stark shift becomes linear.

\section{A molecular fountain}

The Stark shift can be regarded as the potential energy of the molecule in the electric field. Therefore, in an inhomogeneous electric field a mechanical force, $\mathbf{F}=-\nabla W$, acts on a polar molecule. We will use this force to decelerate and cool the molecules. Fig. 4 shows a schematic of the molecular fountain. The fountain consists of three differentially pumped vacuum chambers. In the first chamber, a pulsed beam is formed by expanding a mixture of ammonia and xenon through a small hole into vacuum. By cooling the valve housing to $-70^{\circ} \mathrm{C}$, the average velocity of the beam is lowered to $300 \mathrm{~m} / \mathrm{s}$. The translational temperature of the beam is below $1 \mathrm{~K}$ and the rotational temperature is below $5 \mathrm{~K}$. The most intense part of the beam passes through a $2 \mathrm{~mm}$ skimmer into the second chamber where the molecules are decelerated using a series of pulsed electric field stages. Each stage consists of two parallel cylindrical metal rods, one being connected to a positive and one to a negative switchable high voltage power supply. A molecule in a state with a positive Stark shift will gain Stark energy when entering an electric field. This gain in potential energy will be compensated by a loss in kinetic energy, i.e., the molecule will slow down when entering the field. When we switch off the electric field before the molecule has left the field, the molecule will not regain the lost kinetic energy, and it will remain at its lower velocity. The amount of energy taken from the molecule depends on the magnitude of the Stark shift in the applied electric field. By applying a voltage difference of up to $20 \mathrm{kV}$ an electric field of about $100 \mathrm{kV} / \mathrm{cm}$ is created which results in a Stark shift of $1.0 \mathrm{~cm}^{-1}$ (see Fig. 3). Using 100 pulsed electric field stages, the molecules are decelerated from $300 \mathrm{~m} / \mathrm{s}$ to about $4 \mathrm{~m} / \mathrm{s}$. At the exit of the decelerator the decelerated bunch of molecules has a volume of approximately $1 \mathrm{~mm}^{3}$ and a temperature of about $30 \mathrm{mK}[22]$.

During the deceleration process, the product of the position and velocity spread - the phase space volume - of the decelerated molecules remains constant. We can decrease the velocity spread by increasing the position spread - a process known as adiabatic cooling - or vice versa, decrease the position spread by increasing the velocity spread - known as adiabatic compression or bunching [23]. Both processes are incorporated in the design of the molecular fountain. For this, a combination of two linear quadrupoles and a cylindrical quadrupole is used. The linear quadrupoles are composed of four $20 \mathrm{~mm}$ diameter rods which are placed symmetrically on the outside of a circle with a diameter of $20 \mathrm{~mm}$. By applying a voltage difference of $10 \mathrm{kV}$ between adjacent electrodes an electric field is created which is zero at the center and increases linearly away from the center to a maximum of $10 \mathrm{kV} / \mathrm{cm}$ at the electrodes. As the Stark shift of the $|J, K\rangle=|1,1\rangle$ state of $\mathrm{NH}_{3}$ is quadratic in these fields, molecules in this state will experience 


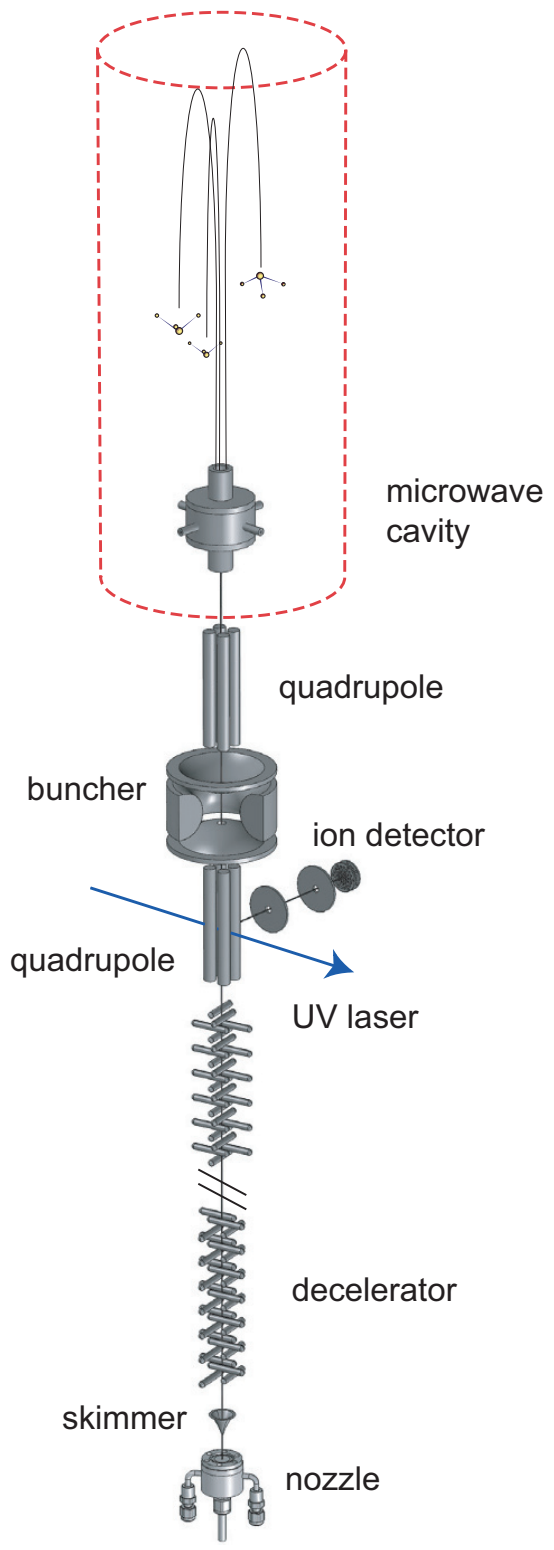

Fig. 4. Schematic view of the molecular fountain currently under construction at the Laser Centre VU in Amsterdam.

a harmonic force towards the molecular beam axis. The two linear quadrupoles are used to expand and cool the beam in the transverse direction, the cylindrical quadrupole does the same in the vertical direction. After passing the microwave cavity, the molecules fly up some $30 \mathrm{~cm}$ more before falling back under gravity. Using again the quadrupole lens system, molecules in the upper inversion level are focused into the lower linear quadrupole. Here molecules in the upper inversion level of the $|J, K\rangle=|1,1\rangle$ state of $\mathrm{NH}_{3}$ are stated-selectively ionized in the focus of a tunable UV laser using $(2+1)$ Resonance Enhanced Multi-Photon Ionization through the $\tilde{B}^{1} E^{\prime \prime}$-state [24]. The resulting ions are mass-selectively detected using the quadrupole electrodes as extraction electrodes in a Wiley-McLaren type mass spectrometer set-up.

Fig. 5 shows the simulated trajectories of some ammonia molecules through the quadrupole focusing system. The exit of the decelerator is taken as $z=0$, and the grey shaded areas show the position of the quadrupoles. On the right side of the figure, the envelope of the beam 


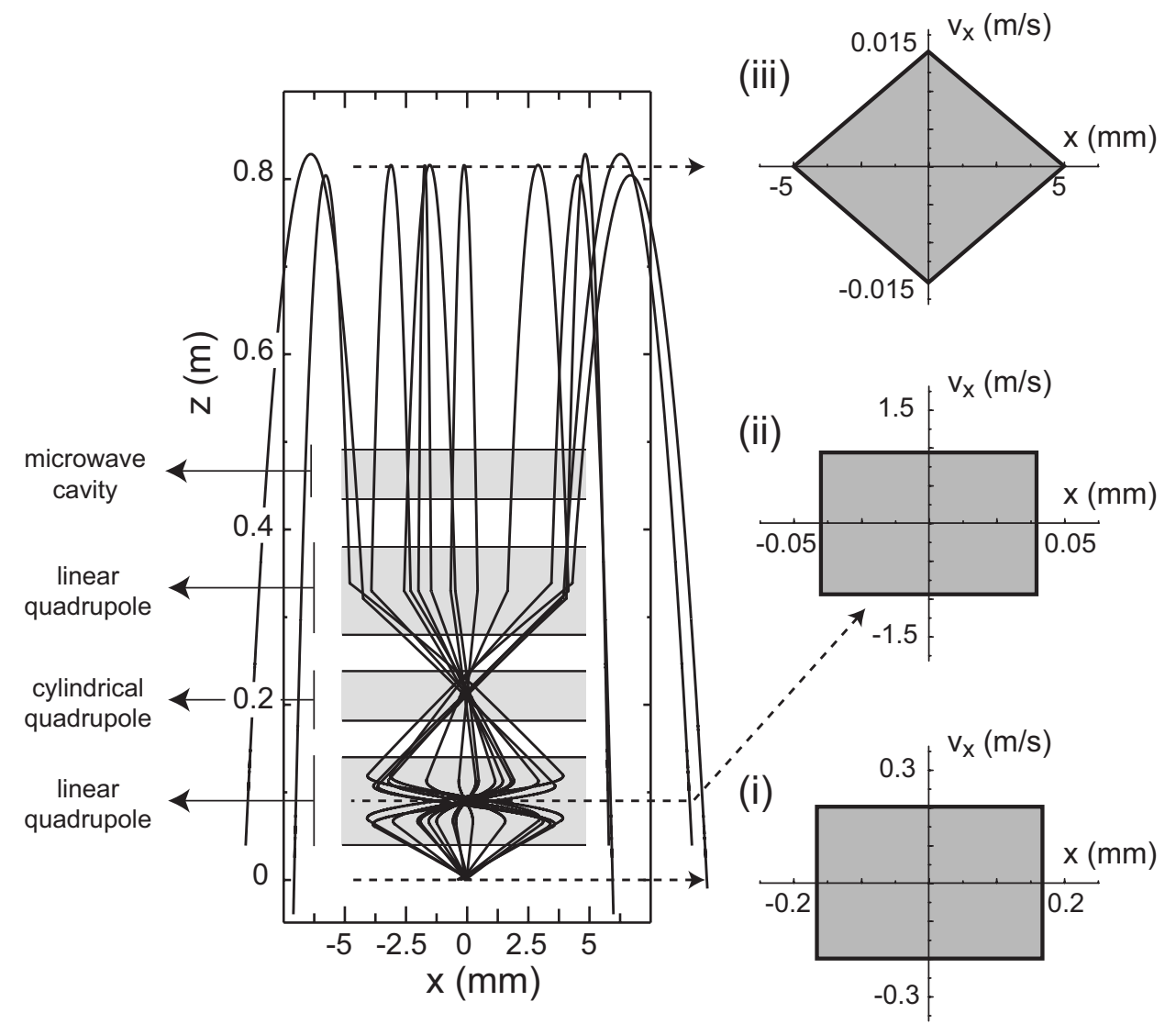

Fig. 5. Simulated trajectories of ammonia molecules through a quadrupole lens system. On the right the phase space distributions of the beam are shown a three different positions in the fountain. Note that the scales of the three graphs are very different.

is plotted in phase space at three positions in the fountain: (i) at the exit of the decelerator (ii) at the mid-point of the first linear quadrupole where they are detected, and (iii) at the apogee of the fountain. Note that the scales of the three graphs are very different. Only molecules that make it through the fountain without hitting the $10 \mathrm{~mm}$ aperture of the microwave cavity are plotted. Therefore, the area bounded by the bold lines represents the transverse acceptance of the fountain. As the trajectories are fully symmetric around the apogee, the phase-space distributions refer to both the up- and down-going beam. With the settings used in the simulation, a $4: 1$ image is made from the exit of the decelerator onto the detection region and a 1 : 100 image is made from the detection region to the apogee of the fountain. In this way the beam has a low temperature $\left(0.015 \mathrm{~m} / \mathrm{s}\right.$ corresponds to $\left.\mathrm{E}_{k i n} / \mathrm{k}_{b}=230 \mathrm{nK}\right)$ and a low density in the interaction region, and a relatively high density and temperature $(1 \mathrm{~m} / \mathrm{s}$ corresponds to $\mathrm{E}_{k i n} / \mathrm{k}_{b}=1 \mathrm{mK}$ ) in the detection region. Using the cylindrical quadrupole, a similar imaging is performed along the $z$-axis. From simulations taking into account the true force (including small non-linear terms) on the molecules, we find the acceptance - the phase space volume occupied by the molecules that make it through the fountain - to be $0.005(\mathrm{~mm} \cdot \mathrm{m} / \mathrm{s})^{3}$. This should be compared to the phase space density of the beam that exits the decelerator. A single decelerated bunch contains approximately $10^{6}$ molecules in a volume of $1 \mathrm{~mm}^{3}$ having a velocity spread of $5 \mathrm{~m} / \mathrm{s}$. Therefore, about 40 molecules are expected to be focused back onto the detection region per fountain cycle where they are detected with near unity efficiency. Here, we have assumed a microwave cavity with an aperture of $10 \mathrm{~mm}$. The acceptance scales with $d^{4}$, with $d$ the aperture of the microwave cavity. There is a trade off between the count rate and errors that are introduced when the hole in the microwave cavity becomes larger (see section 4.2.5). With a careful design of the cavity it might be possible to use a larger aperture. 


\section{Evaluation of stability and accuracy}

A frequency standard is characterised by its stability and accuracy. The stability, tells us the statistical uncertainty of a frequency measurement as function of measurement time. The accuracy tells us how well the measured frequency agrees with the unperturbed molecular frequency. On short time scales, the frequency uncertainty is determined by the stability, on long time scales the uncertainty is determined by the accuracy. Note that for tests of the time-variation of the proton-electron ratio, discussed in section 5, systematic effect are only relevant when they change over time. For instance, shifts due to blackbody radiation are unimportant as long as the measurements are always taken at the same temperature.

\subsection{Stability}

A measure of the fractional instability is provided by the Allen variance [25],

$$
\sigma_{y}(\tau)=\frac{1}{Q} \frac{1}{\sqrt{N \tau / \tau_{c}}},
$$

where $Q$ is the quality factor of the transition given by the transition frequency over the measured linewidth, $f / \Delta f, N$ is the number of ions detected per cycle, $\tau$ is the measurement time and $\tau_{c}$ is the duration of a single cycle. If we assume an interaction time of $0.5 \mathrm{~s}$, the measured linewidth will be about $1 \mathrm{~Hz}$, and the Q-factor is about $2 \times 10^{10}$. For transitions originating from the $F^{\prime}=1, F_{1}^{\prime}=0.5$ (7) state, we expect to detect about 30 ions per cycle. As a single cycle takes 0.8 seconds, we find $\sigma_{y}=7 \times 10^{-12} \tau^{-1 / 2}$. This implies that a measurement time of about 1.5 hours is required to reach an accuracy of $1 \times 10^{-13}$, and 150 hours to reach an accuracy of $1 \times 10^{-14}$.

\subsection{Accuracy}

From the $F^{\prime}=1, F_{1}^{\prime}=0.5(7)$ and the $F^{\prime}=0, F_{1}^{\prime}=0.5$ (8) levels, six transitions to the lower inversion level are possible. These transitions are shown in Fig. 2. From these transitions the $F^{\prime}=1, F_{1}^{\prime}=0.5 \leftrightarrow F^{\prime \prime}=1, F_{1}^{\prime \prime}=0.8(7 \leftrightarrow 2)$ transition at $22624941.08 \mathrm{kHz}$ offers the highest accuracy, while the other transitions may be used to monitor and correct for stray electric and magnetic fields in the interrogation zone. Using the REMPI scheme, molecules in all low-field seeking states of the $|J, K\rangle=|1,1\rangle$ state of $\mathrm{NH}_{3}$ will be ionized. To avoid background signal due to molecules in level 8 , we need to implement a second microwave cavity driving molecules in level 8 to a high-field seeking state, or, alternatively, use rf-radiation resonant with the $8 \rightarrow 6$ transition.

We have evaluated all known sources of error that limit the accuracy in the fountain. The accuracy budget for the $7 \leftrightarrow 2$ transition is listed in Table 1 . All systematic effects are well known and well studied in atomic fountains $[1,2]$ but need to be re-evaluated for molecules. In what follows the most bothersome frequency shifts will be discussed.

\subsubsection{Collisional shift}

When molecules get close to one another, their levels will shift due to intermolecular interactions. As the shift of the upper level is generally different from that of the lower level, a collision will result in a phase shift. The accumulation of phase shifts introduced by each collision produces an average frequency shift of the molecular resonance. This collision shift is given by $[25]$ :

$$
\Delta f_{c}=n v \sigma_{c}
$$

with $n$ the molecular density, $v$ the mean relative velocity, and $\sigma_{c}$ the cross section of the collision shift. In the absence of an electric field, ammonia molecules are not oriented and there is 
Table 1. The accuracy budget for the $F^{\prime}=1, F_{1}^{\prime}=0.5 \leftrightarrow F^{\prime \prime}=1, F_{1}^{\prime \prime}=0.8(7 \leftrightarrow 2)$ inversion transition in the $|J, K\rangle=|1,1\rangle$ state of ${ }^{15} \mathrm{NH}_{3}$ at $22624941.08 \mathrm{kHz}$.

\begin{tabular}{|c|c|c|c|}
\hline Contributor & $\begin{array}{l}\text { Shift } \\
\left(\times 10^{15}\right) \\
\Delta f / f\end{array}$ & $\begin{array}{l}\text { Uncertainty } \\
\left(\times 10^{15}\right) \\
\Delta f / f\end{array}$ & \\
\hline Collisions & $<1$ & $<1$ & $\begin{array}{l}\text { If temperature of launched } \\
\text { molecules }<1 \mathrm{mK} \\
\& \text { density }<1 \times 10^{5} / \mathrm{cm}^{3}\end{array}$ \\
\hline Zeeman effect & $<1$ & $<1$ & If magnetic field $<0.1 \mathrm{mG}$ \\
\hline Stark effect & $<1$ & $<1$ & If electric field $<1 \mathrm{mV} / \mathrm{cm}$ \\
\hline $\begin{array}{l}\text { Blackbody radiation } \\
\text { Room temperature } \\
77 \mathrm{~K}\end{array}$ & $\begin{array}{l}-190 \\
-20\end{array}$ & $\begin{array}{l}19 \\
2\end{array}$ & Uncertainty caused by the calculations \\
\hline $\begin{array}{l}1^{\text {st }} \text { order Doppler } \\
2^{\text {nd }} \text { order Doppler \& } \\
\text { Gravitational red shift } \\
\text { Pulling by other transitions }\end{array}$ & $\begin{array}{c}- \\
<1\end{array}$ & $\begin{array}{l}<2 \\
<0.1 \\
<1\end{array}$ & If altitude known to $\pm 0.1 \mathrm{~m}$ \\
\hline
\end{tabular}

no dipole-dipole interaction. Therefore, the interactions are dominated by the electric dipoleinduced dipole interaction [26], i.e., the shift of an ammonia molecule is induced by the dipole moment of its collision partner. This Stark shift is opposite for molecules in the upper and lower inversion level of ammonia. Fig. 6 shows the dependence of $\sigma_{c}$ as a function of kinetic energy for both ${ }^{15} \mathrm{NH}_{3}$ and ${ }^{14} \mathrm{NH}_{3}$ using the model for the electric dipole-induced dipole interaction described in ref. [26]. For ${ }^{15} \mathrm{NH}_{3}$, which is a Fermion, only even partial scattering waves contribute, while for ${ }^{14} \mathrm{NH}_{3}$, which is a Boson, only odd partial scattering waves contribute. For energies below $100 \mu \mathrm{K}$ the fractional frequency shift in ${ }^{15} \mathrm{NH}_{3}$ becomes equal to $2 \times 10^{-20}$ times the density in $\mathrm{cm}^{-3}$, independent of temperature. From the discussion in section 3 , we estimate the density in the interaction zone to be $n \sim 5 \times 10^{4} \mathrm{~cm}^{-3}$, hence $\Delta f_{c} / f$ is below $10^{-15}$.

\subsubsection{Blackbody radiation}

At non-zero temperature, the walls of the surrounding vacuum chambers continuously emit blackbody radiation. This blackbody radiation couples the upper and lower inversion levels to higher rotational and vibrational levels causing AC-Stark shifts. As transitions from the upper and lower inversion frequency have slightly different frequencies the two inversion levels experience a different shift. This implies that the transition measured at non-zero temperature is shifted with respect to the unperturbed $(T=0 \mathrm{~K})$ value. At room temperature the spectral energy density of the blackbody radiation at frequency $f$ is given by:

$$
\rho(f)=\frac{8 \pi h f^{3}}{c^{3}} \frac{1}{\exp \left(h f / k_{B} T\right)-1},
$$

with $T$ the temperature of the surrounding walls. At room temperature the blackbody spectrum peaks at a frequency around $600 \mathrm{~cm}^{-1}$. For atoms, this frequency is far away from all resonance frequencies and the blackbody radiation can be effectively treated as a static electric field [27]. Polar molecules, on the other hand, have strong rotational and vibrational transitions in the infrared region of the spectrum which need to be considered [28,29]. The frequency shift, $\Delta f_{B}$ induced by blackbody radiation is given by:

$$
\Delta f_{B}=\frac{2}{3 \varepsilon_{0} h^{2}}\left(\sum_{a \rightarrow i} \int d f \rho(f) \frac{\mu_{a \rightarrow i}^{2}}{f-f_{a \rightarrow i}}-\sum_{s \rightarrow i} \int d f \rho(f) \frac{\mu_{s \rightarrow i}^{2}}{f-f_{s \rightarrow i}}\right),
$$




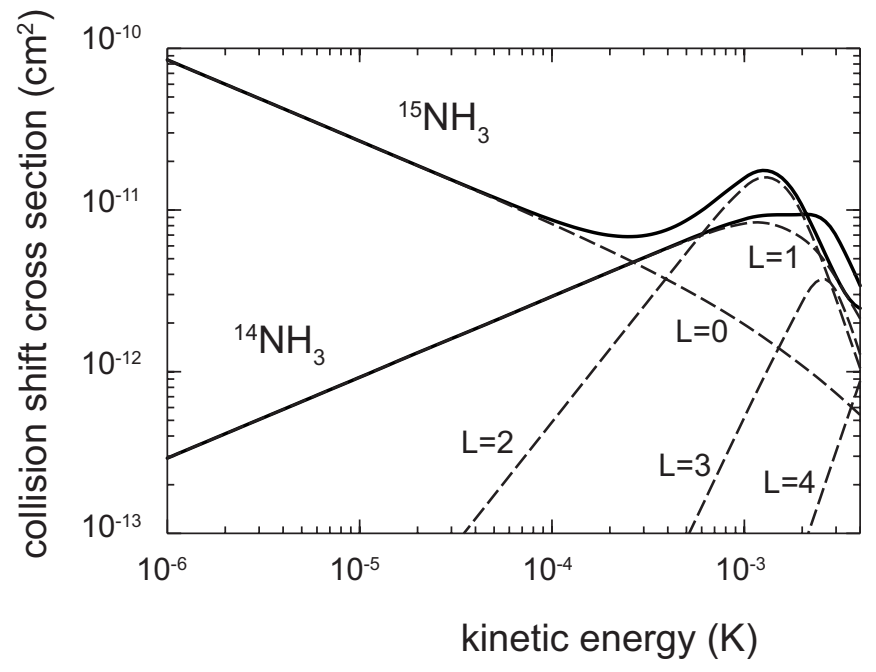

Fig. 6. The collision shift cross section of ${ }^{15} \mathrm{NH}_{3}$ and ${ }^{14} \mathrm{NH}_{3}$ in the $J=1, K=1$ level as a function of kinetic energy.

with $\mu_{s \rightarrow i}$ and $\mu_{a \rightarrow i}$, the transition dipole moment from the symmetric or anti-symmetric level to a state $i$, respectively and $f_{s \rightarrow i}$ and $f_{a \rightarrow i}$, the transition frequencies to this state. The summation should run over all accesible states. The largest contribution comes from the following couplings:

$$
\begin{aligned}
(J=1, K=1, a) & \leftrightarrow(J=1, K=1, s), \\
(J=1, K=1, a) & \leftrightarrow(J=2, K=1, s), \\
(J=1, K=1, s) & \leftrightarrow(J=2, K=1, a), \\
\left(\nu_{2}=0, J=1, K=1, a\right) & \leftrightarrow\left(\nu_{2}=1, J=1,2, K=1, s\right), \\
\left(\nu_{2}=0, J=1, K=1, s\right) & \leftrightarrow\left(\nu_{2}=1, J=1,2, K=1, a\right),
\end{aligned}
$$

with $\nu_{2}$ the umbrella vibrational motion. Fig. 7 shows the results of Eq. (6) considering taking only into account these 5 transitions. The transition dipole moments are taken from Urban et al. [30]. At $300 \mathrm{~K}$, the fractional blackbody shift is found to be $1.9 \times 10^{-13}$ (with an estimated precision of 10\%). Temperature fluctuations cause frequency shifts of $d\left(\Delta f_{B} / f\right) / d T=-8 \times 10^{-16} / \mathrm{K}$. These numbers are in agreement with a recent calculation by Vanhaecke and Dulieu [29].

\subsubsection{Stark shift}

As discussed in section 2, the large dipole moment of ammonia induces a large shift in an electric field, which is opposite for both inversion levels. In Fig. 3(b) the Stark shift is shown in low electric fields. As the different $M_{f}$ components have a different Stark shift the $7 \leftrightarrow 2$ transition will both be broadened and shifted. In fields below $1 \mathrm{~V} / \mathrm{cm}$ all different $\Delta M_{F}$ transitions will overlap. The Stark shift of the linecenter is equal to:

$$
\Delta f_{S}=7.3 \mathrm{~Hz} /(\mathrm{V} / \mathrm{cm})^{2} .
$$

If the electric field is kept below $1 \mathrm{mV} / \mathrm{cm}, \Delta f_{S} / f$ is below $10^{-15}$.

\subsubsection{Zeeman shift}

In the ground state, ammonia has no unpaired electrons. The Zeeman shift is due to the interaction of the external magnetic field with the nuclear spin of the ${ }^{15} \mathrm{~N}$ nucleus and with 


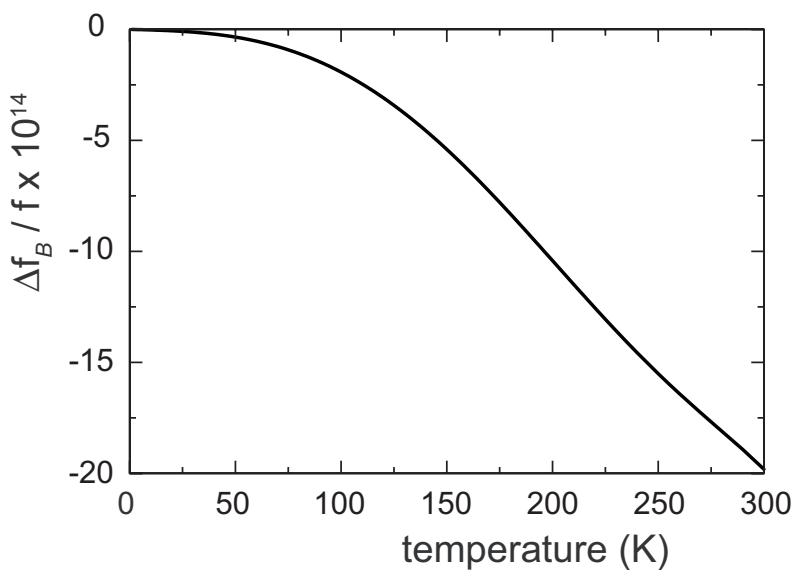

Fig. 7. Blackbody shift of the inversion frequency in the $J=1, K=1$ level of ${ }^{15} \mathrm{NH}_{3}$ as a function of the temperature of the setup.
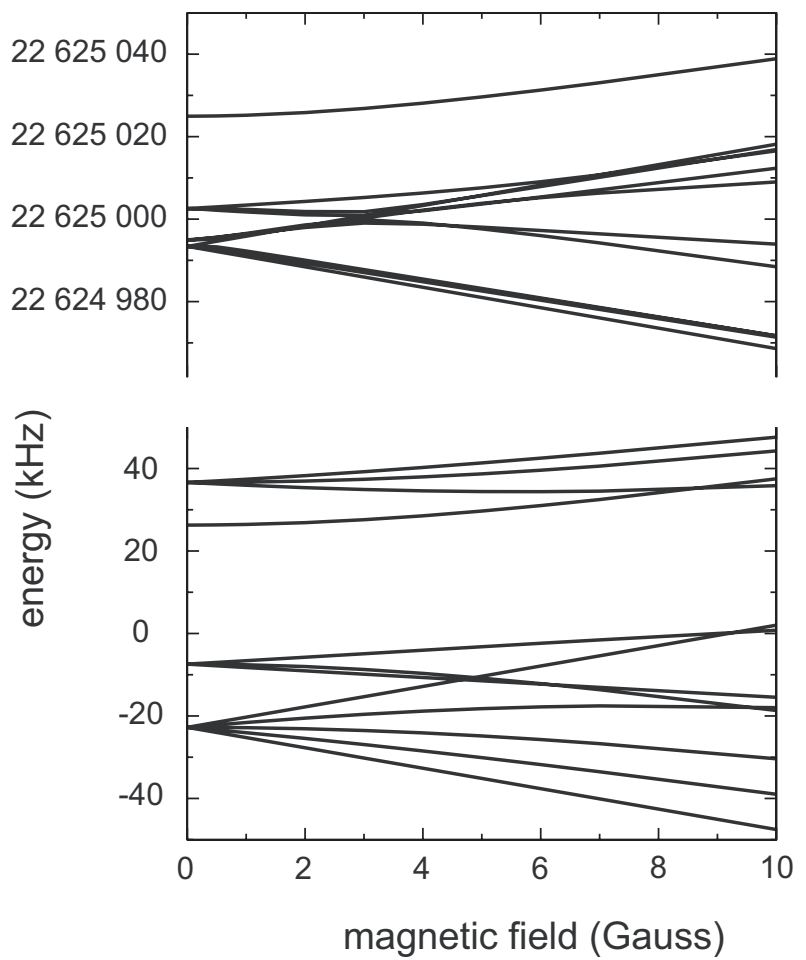

Fig. 8. Zeeman shift of the anti symmetric (upper graph) and symmetric (lower graph) component of the $J=1, K=1$ level in ${ }^{15} \mathrm{NH}_{3}$.

the nuclear spins of the hydrogen atoms. In Fig. 8 the Zeeman shift is shown in magnetic fields up to 1 Gauss. For comparison, the Zeeman shift in Cesium atoms is 4 orders in magnitude larger. In cesium fountain clocks a strong bias field, the so-called $C$-field, is applied to shift all transitions far away from the $F=4, M_{F}=0 \leftrightarrow F=3, M_{F}=0$ clock transition. For ammonia, a bias field is neither necessary nor practical. If the magnetic field is below $1 \mathrm{mG}$, all different $\Delta M_{F}$ transitions of the $7 \leftrightarrow 2$ transition will overlap. The Zeeman shift of the linecenter is equal to:

$$
\Delta f_{Z}=-16 \mathrm{~Hz} / \mathrm{G}^{2} .
$$


If the magnetic field is kept below $0.1 \mathrm{mG}, \Delta f_{Z} / f$ is below $10^{-15}$. Note that, the $7 \leftrightarrow 3$ transition has a 10 times larger Zeeman shift and can be used to monitor and correct for stray magnetic fields.

\subsubsection{First order Doppler effect}

Launched molecules interact with the microwave twice, as they fly up and as they fall back. In general, the position where a molecule passes the cavity the first time will be different from the position where it passes the cavity the second time. We will call these positions $A$ and $B$. If the phase of the microwave field at position $A$ and position $B$ is not the same, the transition will be Doppler shifted. This shift is given by:

$$
\Delta f_{F D}=\frac{d \xi(B)-d \xi(A)}{2 \pi T_{R}}
$$

where $\delta \xi(A)$ and $\delta \xi(B)$ are the phase distributions at position $A$ and $B$, and $T_{R}=2 v_{0} / g$ is the time interval between the two passes through the cavity. To induce the inversion transition in ammonia, we will use the $\mathrm{TM}_{011}$ mode of a cylindrical microwave cavity. Using a cavity that is fed from two sides, the phase inside the cavity is approximately given by [31]:

$$
\delta \xi \approx\left(\frac{r}{a}\right)^{2} \cos (2 \phi) \frac{\pi}{24 Q_{c a v}}
$$

where $Q_{c a v}$ is the $Q$-value and $a$ the radius of the cavity. Assuming $Q_{c a v}=1000, a=28 \mathrm{~mm}$, and $r_{\max }=5 \mathrm{~mm}, \delta \xi(B)-d \xi(A)$ is below $40 \mu \mathrm{rad}$ and $\Delta f_{F D} / f$ is below $2 \times 10^{-15}$. This is an overestimation as the symmetry of the trajectories will cause a partial cancellation of the effect; the shift of a molecule passing the cavity the first time at position $A$ and the second time at position $B$ will be cancelled by the shift of a molecule passing the cavity the first time at position $B$ and the second time at position $A$.

\subsubsection{Relativistic effects}

Frequency shifts are induced by relativistic effects, i.e., by the second order Doppler shift and by gravitational red shift. In a fountain these are given by [25]:

$$
\begin{aligned}
\frac{\Delta f_{R}}{f} & =\frac{g}{2 v_{0}} \int_{0}^{2 v_{0} / g}\left[-\frac{\left(-g t+v_{0}\right)^{2}}{2 c^{2}}+\frac{g}{c^{2}}\left(-\frac{g t^{2}}{2}+v_{0} t\right)\right] d t+\frac{g h_{0}}{c^{2}} \\
& =\frac{g h_{0}}{c^{2}}-\frac{v_{0}^{2}}{3 c^{2}}
\end{aligned}
$$

where $h_{0}$ is the altitude of the microwave cavity compared to the local geodesic and $v_{0}$ is the velocity of the molecules as they pass through the microwave cavity. Taking $v_{0}=3 \mathrm{~m} / \mathrm{s}$, the second term of equation (11) is less than $10^{-16}$. If the value of $h_{0}$ is measured within an uncertainty of $0.1 \mathrm{~m}$, also the first term of equation (11) is below $10^{-16}$.

\subsubsection{Pulling effects}

Neighbouring transitions in the microwave spectrum will cause frequency shifts. The shift due to these pulling effects, $\Delta f_{p}$, is given by [25]:

$$
\Delta f_{p}=\sum_{i} \frac{1}{2(2 \pi)^{3}} \frac{\Delta I_{i}}{T_{R}^{2} \tau_{R}^{2} \Delta f_{i}^{3}}
$$




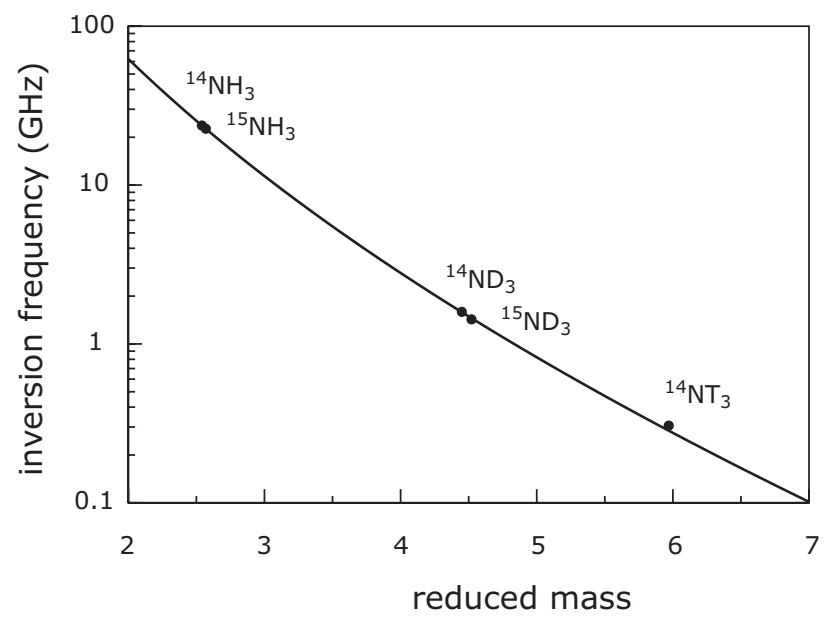

Fig. 9. Inversion frequency in the $J=1, K=1$ state for the various isotopomers of ammonia as a function of the reduced mass of the $\nu_{2}$ vibration. The solid lines shows the result of a simple model [37].

where $\Delta f_{i}$ is the frequency difference between the neighbouring line and clock transition. $\Delta I_{i}$ is the intensity of the additional line relative to the clock transition. $T_{R}=2 v_{0} / g$ is the time interval between the two passes through the cavity and $\tau_{R}=l_{c} / v_{0}$ is the time the molecules spend in the cavity. All neighbouring transitions to the $7 \leftrightarrow 2$ transition are more than $10 \mathrm{kHz}$ displaced. Further, taking $T_{R}=0.5 \mathrm{~s}, \tau_{R}=0.01 \mathrm{~s}$ and $\Delta I_{i}=1, \Delta f_{p} / f$ is below $10^{-20}$.

\section{Sensitivity of the inversion frequency in ammonia to a time-variation of the proton-electron mass ratio}

Recent astrophysical data suggest that the fine-structure constant, $\alpha$, and the proton-electron mass ratio, $m_{p} / m_{e}$, [32,33] may have changed over the past several billion years. If confirmed, this would be a major scientific breakthrough with profound implications on how we understand the universe. Therefore, a large number of laboratory tests are currently being performed to ascertain these results (for a recent overview see the paper by Peik and Karshenboim [34] in this volume). Comparisons between atomic frequency standards are sensitive to a possible variation of $\alpha$. Molecular frequency standards, on the other hand, are sensitive to a possible time-variation of $m_{p} / m_{e}$. This is of particular interest as theoretical models predict that the $m_{p} / m_{e}$ variation could be significantly larger than the variation of $\alpha$. Calmet and Fritsch, for instance, predict that the variation of $m_{p} / m_{e}$ is 36 times larger than the variation of $\alpha$ [35]. Molecules can also be used to test the time dependence of the ratio of the mass of different nuclei or the proton-neutron mass ratio, which currently have not been tested at levels better than $10^{-12} / \mathrm{yr}$. These tests are also tests of the time-independence of QCD interaction through the mass scale $\Lambda_{\mathrm{QCD}}[36]$.

Since the inversion mode of ammonia involves tunneling of the nitrogen atom through the plane formed by the hydrogen atoms (or vice versa) its frequency is very sensitive to the mass of the proton. This is illustrated by the fact that the inversion frequency of ${ }^{15} \mathrm{NH}_{3}$ changes from $22.6 \mathrm{GHz}$ to $1.43 \mathrm{GHz}$ when the hydrogen atoms are replaced by deuterium atoms. This implies that if the proton mass (or rather the ratio of the proton mass to the electron mass) shifts over time, so does the inversion frequency. In Fig. 9 the frequencies for the different ammonia isotopomers are plotted as a function of their reduced mass [7,17-19,37] (note the log scale of the vertical axis). Theoretically [37] one expects the inversion frequency to follow the formula

$$
f=\frac{a_{0}}{\sqrt{m}} e^{-a_{1} \sqrt{m}}
$$


with $m$ the reduced mass of ammonia for the umbrella vibration in amu and $a_{0}$ and $a_{1}$ constants. The solid line in Fig. 9 shows a fit taking $a_{0}=68 \mathrm{THz} \times \mathrm{amu}^{1 / 2}$ and $a_{1}=4.7 \mathrm{amu}^{-1 / 2}$. From this, we can determine the sensitivity of the inversion frequency to changes in $m_{p} / m_{e}$. For ${ }^{15} \mathrm{NH}_{3}$, we find $\delta f / f=-4.2 \delta\left(m_{p} / m_{e}\right) /\left(m_{p} / m_{e}\right)$. For comparison, 'ordinary' vibrational transitions have a sensitivity of $\delta f / f=-0.5 \delta\left(m_{p} / m_{e}\right) /\left(m_{p} / m_{e}\right)$; the sensitivity of the inversion frequency to a variation of $m_{p} / m_{e}$ is enhanced by almost a factor of ten. Note that here we assume that the neutron mass changes the same way as the proton mass. If a time-variation of $m_{p} / m_{e}$ would be found, measurements in $\mathrm{NH}_{3}$ and $\mathrm{ND}_{3}$ can be used to check the validity of this assumption.

\section{Conclusions}

The recent demonstration of cooling and manipulation techniques for molecules offer new possibilities for precision measurements in molecules. Here, we have presented the design of a molecular fountain based on a Stark decelerated molecular beam. By using a combination of two linear electric quadrupoles and a cylindrical electric quadrupole, the beam is cooled to sub-microKelvin temperatures. Based on simulations of the trajectories through the fountain we expect to detect about 30 ions per fountain cycle, which translates in a frequency instability of $\sigma_{y}=7 \times 10^{-12} \tau^{-1 / 2}$, with $\tau$ being the measurement time in seconds. Once the time sequence used to trigger the voltages on the decelerator and the focusing system is optimised, the machine should be able to run virtually unattended. This will allow continuous data taking over extended periods in time. We have evaluated all possible sources of systematic frequency shifts. Compared to Cesium, ammonia is particularly sensitive to shifts due to cold collisions and due to blackbody radiation. This is probably generally true for molecules. As the anticipated densities in the molecular fountain are relatively small, cold collision shifts will not be a problem. In order to reach accuracies below $10^{-14}$, more accurate calculations are needed to determine the shift due to blackbody radiation. With the anticipated stability, a fractional accuracy of $10^{-14}$ requires a measurement time of 150 hours. This corresponds to an absolute accuracy of $0.2 \mathrm{mHz}$, a 10000 fold improvement over the current most precise microwave measurement in a molecule. If this measurement is repeated after 2 years, the variation of the proton-electron mass ratio can be constrained at the level of $10^{-15}$ per year.

We thank Ruth Buning and Jacqueline van Veldhoven for useful discussions. H.L.B acknowledges financial support from the Netherlands Organisation for Scientific Research (NWO) via a VENI-grant.

\section{References}

1. S. Bize, et al., J. Phys. B. - Atom. Mol. Opt. Phys. 38, S449 (2005)

2. T.P. Heavner, et al., Metrologia 42, 411 (2005)

3. M.M. Boyd, et al., Phys. Rev. Lett. 98, 083002 (2007)

4. W.H. Oskay, et al., Phys. Rev. Lett. 97, 020801 (2006)

5. H.L. Bethlem, G. Meijer, Int. Rev. Phys. Chem. 22, 73 (2003)

6. J. Doyle, B. Friedrich, R.V. Krems, F. Masnou-Seeuws, Eur. Phys. J. D 31, 149 (2004)

7. J. van Veldhoven, J. Küpper, H.L. Bethlem, B. Sartakov, A.J. van Roij, G. Meijer, Eur. Phys. J. D 31, 337 (2004)

8. E.R. Hudson, H.J. Lewandowski, B.C. Sawyer, J. Ye, Phys. Rev. Lett. 96, 143004 (2006)

9. J.J. Hudson, B.E. Sauer, M.R. Tarbutt, E.A. Hinds, Phys. Rev. Lett. 89, 023003 (2002)

10. D. Kawall, F. Bay, S. Bickman, Y. Jiang, D. DeMille, Phys. Rev. Lett. 92, 133007 (2004)

11. D. DeMille, S.B. Cahn, D. Murphree, D.A. Rahmlow, M.G. Kozlov, Phys. Rev. Lett. 100, 023003 (2008)

12. Ch. Daussy, et al., Phys. Rev. Lett. 83, 1554 (1999)

13. A. Shelkovnikov, R.J. Butcher, C. Chardonnet, A. Amy-Klein, Phys. Rev. Lett. 100, 150801 (2008)

14. V.V. Flambaum, Eur. Phys. J. Special Topics 163, 159 (2008)

15. P. Foreman, Proc. IEEE 73, 1181 (1985) 
16. J.P. Gordon, H.J. Zeiger, C.H. Townes, Phys. Rev. 99, 1264 (1955)

17. S.G. Kukolich, Phys. Rev. 156, 83 (1967)

18. J.T. Hougen, J. Chem. Phys. 156, 83 (1972)

19. J. van Veldhoven, R.T. Jongma, B. Sartakov, W.A. Bongers, G. Meijer, Phys. Rev. A 66, 032501 (2002)

20. S. Urban, J. Quant. Spec. Rad. Trans. 48, 675 (1992)

21. C.H. Townes, A.L. Schawlow, Microwave Spectroscopy (McGraw-Hill, New York, 1955)

22. H.L. Bethlem, F.M.H. Crompvoets, R.T. Jongma, S.Y.T. van de Meerakker, G. Meijer, Phys. Rev. A 65, $053416(2002)$

23. F.M.H. Crompvoets, R.T. Jongma, H.L. Bethlem, G. Meijer, Phys. Rev. Lett. 89, 093004 (2002)

24. M.N.R. Ashfold, R.N. Dixon, N. Little, R.J. Stickland, C.M. Western, J. Chem. Phys. 89, 1754 (1988)

25. J. Vanier, C. Audoin, The Quantum Physics of Atomic Frequency Standard (IOP Publishing, Bristol, 1989)

26. M. Kajita, Phys. Rev. A 74, 032710 (2006)

27. W.M. Itano, L.L. Lewis, D.J. Wineland, Phys. Rev. A 25, 1233 (1982)

28. S. Hoekstra, et al,, Phys. Rev. Lett. 98, 133001 (2007)

29. N. Vanhaecke, O. Dulieu, Mol. Phys. 105, 1723 (2007)

30. S. Urban, D. Papousek, V.M. Devi, B. Fridovich, R. D’Cunha, K.N. Rao, J. Mol. Spectrosc. 86, 38 (1984)

31. S.R. Jefferts, R.E. Drullinger, A. DeMarchi, Proc. IEEE Int. Freq. Control Symp. (1998)

32. J.K. Webb, et al., Phys. Rev. Lett. 87, 091301 (2001)

33. E. Reinhold, R. Buning, U. Hollenstein, A. Ivanchik, P. Petitjean, W. Ubachs, Phys. Rev. Lett. 96, $151101(2006)$

34. E. Peik, S. Karshenboim, Eur. Phys. J. Special Topics 163, 1 (2008)

35. X. Calmet, H. Fritzsch, Eur. Phys. J. C 24, 693 (2002)

36. J.-P. Uzan, Rev. Mod. Phys. 75, 403 (2003)

37. P. Helminger, F.C. DeLucia, W. Gordy, H.W. Morgan, P.A. Staats, Phys. Rev. A 9, 12 (1974) 